\title{
Measuring $\alpha-Z r$ grain Size in extruded Zr-2.5Nb Pressure Tubes by using linear intercept lengths from SEM images
}

\author{
A.J. Lockley* R.E. Mayville** \\ *Materials \&Mechanics Branch, **Deformation Technology Branch, \\ Chalk River Laboratories, Atomic Energy of Canada, Chalk River, ON. K0J 1J0
}

Pressure tubes made from $\mathrm{Zr}-2.5 \mathrm{Nb}$ are a significant component of CANDU reactors [1]. The ability to determine the size of $\alpha-\mathrm{Zr}$ grains in this material is necessary so that correlation of general microstructure with behaviour of mechanical properties, such as creep resistance and fracture toughness, can be made to increase component life and reliability. In order to determine the $\alpha-\mathrm{Zr}$ grain size distribution a method involving careful specimen preparation, image processing, and computer assisted measuring was developed.

The microstructure of a $\mathrm{Zr}-2.5 \mathrm{Nb}$ pressure tube comprises an $\alpha-\mathrm{Zr}$ phase in the form of attenuated platelets partially delineated with a $\mathrm{Nb}$ rich $\beta-\mathrm{Zr}$ phase. The grain elongation is the result of the extrusion process during manufacture and because the grains typically form as Widmanstatten platelets. The $\alpha-Z r$ grains have an average thickness of less than $1 \mu \mathrm{m}$ (in the radial direction) and as such their size cannot be reasonably determined using light microscopy, Figure 1. Conventionally, $\alpha-Z r$ grains have been measured using images produced from TEM replicas where measurements of the width were made manually; one grain at time. Attempts to isolate and measure individual $\alpha-Z r$ grains using automated image analysis were generally unsuccessful because the $\beta-Z r$ phase was too discontinuous to delineate the $\alpha-Z r$ grains. Regardless, an automated method of surveying both a large field of grains and a large number of specimens was required. Therefore, a linear intercept method was adopted using an image analyser to measure the $\alpha-\mathrm{Zr}$ grain images produced from a Scanning Electron Microscopy (SEM).

Success of the method depends greatly on specimen preparation. Specimens are polished to a $1 \mu \mathrm{m}$ finish and etched using a solution comprising 45\% lactic acid, 45\% HNO3, and 5\% $\mathrm{HF}$ that is swabbed on to the prepared surface for a duration of about 10 seconds. The relief primarily arises because the $\alpha-\mathrm{Zr}$ phase dissolves at a faster rate than the $\beta-\mathrm{Zr}$ phase. Care must be taken to etch specimens properly as under etching will not adequately delineate the two phases and over etching deteriorates the $\alpha-Z r$ phase to the extent that the $\beta-Z r$ phase collapses and obscures the $\alpha-Z r$ grains. An over-etched sample is compared with a satisfactory sample in Figure 2 . The microstructure is imaged using SEM and is captured digitally in a low contrast mode to ensure all image data is preserved within 256 grey levels. Images are captured at 2000x magnification, a suitable compromise for maintaining grain resolution and maximizing sampling area. A typical sampling area is about 35 by $35 \mu \mathrm{m}$ per field. Contrast enhancement and image noise reduction is accomplished using image processing and $\alpha-\mathrm{Zr}$ grains are segmented by grey level thresholding. A linear grid with predetermined grid spacing, nominally $2 \mu \mathrm{m}$, is used to determine linear intercepts. A Boolean AND operation combining the grid and the processed $\alpha$ $\mathrm{Zr}$ grain image produces linear segments representing $\alpha-Z r$ grain lengths for both the radial and 
transverse direction of the pressure tube. These data are then analysed to produce summary statistic and grain size distribution information, Figure 3. [2]

\section{References}

[1] CANada Deuterium Uranium, registered trademark of Atomic Energy of Canada Limited.

[2] Thanks to J.C. Owens for excellent sample preparations.

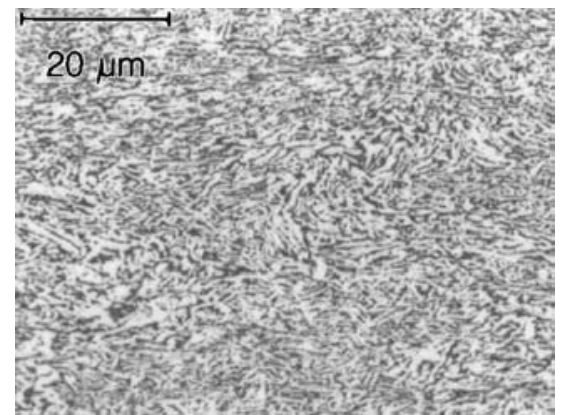

Figure 1: Microstructure of a $\mathrm{Zr}-2.5 \mathrm{Nb}$ pressure tube, using light microscopy, comprising a- $\mathrm{Zr}$ (white) and b-Zr (black) phases.
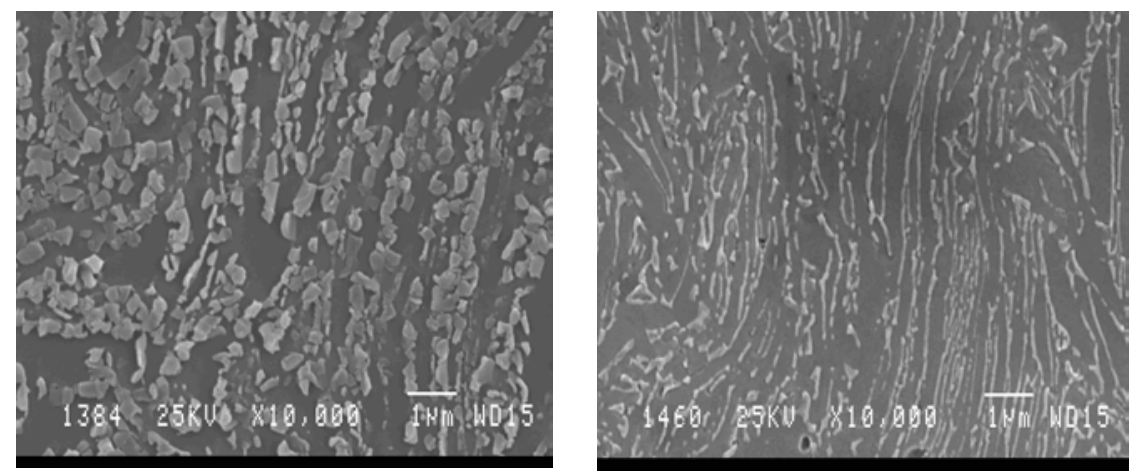

Figure 2: SEM images provide better resolution of the phases: $\alpha-\mathrm{Zr}$ (dark) and $\beta-\mathrm{Zr}$ (light grey). Here, a comparison is made of microstructures that are over-etched, as indicated by the collapsed $\beta$-Zr phase (left), and a properly etched suitable for grain size determination (right).

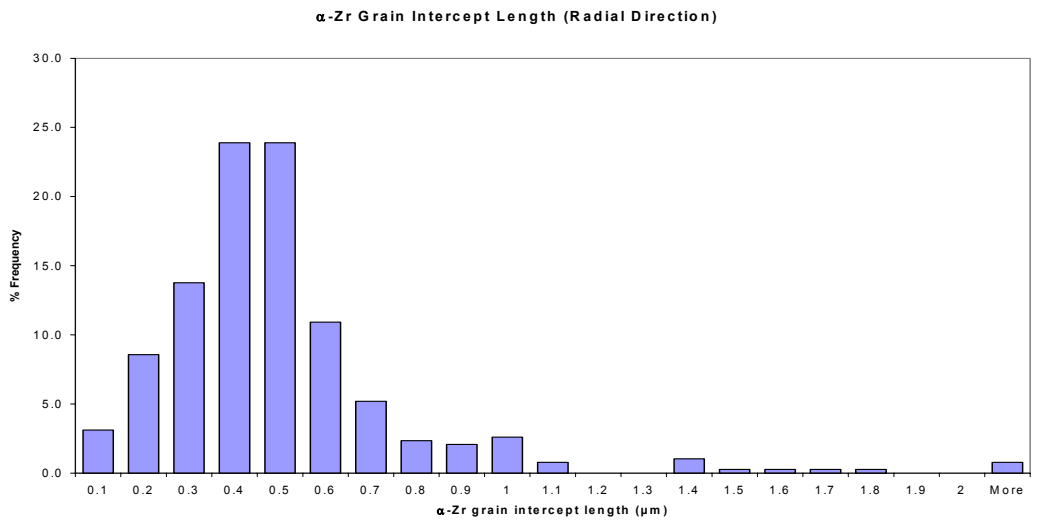

Figure 3: A distribution of $\alpha-Z r$ grain intercept lengths from Figure 2 using automated intercept method. 